\title{
011 - Cor a 14: the missing link in the molecular diagnosis of hazelnut allergy?
}

\author{
Margaretha Faber ${ }^{1,2^{*}}$, Maaike De Graag ${ }^{1,2}$, Catharina Van Der Heijden ${ }^{1,2}$, Vito Sabato ${ }^{1,2}$, Margo M Hagendorens ${ }^{1,2,3}$, \\ Chris H Bridts ${ }^{1,2}$, Luc S De Clerck ${ }^{1,2}$, Didier G Ebo ${ }^{1,2}$ \\ From 3rd Pediatric Allergy and Asthma Meeting (PAAM) \\ Athens, Greece. 17-19 October 2013
}

\section{Background}

Hazelnut (Corylus avellana) allergy shows age and geographic related sensitization profiles that have not been fully resolved.

\section{Objectives}

To study sensitization to hazelnut components in different age groups of hazelnut allergic patients and infants with atopic dermatitis (AD) sensitized to hazelnut in a birchendemic region.

\section{Methods}

Seventy-five hazelnut allergic patients, 14 infants below 1 year of age with $\mathrm{AD}$ sensitized to hazelnut and 15 age related healthy control individuals were tested for IgE reactivity to rCor a 1.04 , rCor a 8 , rCor a 9 , nCor a 11 , rCor a 14 by ImmunoCAP and rBet $\mathrm{v} 1$ by ISAC 103 microarray.

\section{Results}

Thirty-seven patients suffered from a systemic reaction and 38 patients reported an oral allergy syndrome (OAS) after eating hazelnut. In the population with systemic reactions, sensitization to Cor a 14 was seen in $19 / 20$ preschoolchildren (median age (range) 2.6 years $(1.0-5.4)$ ), $8 / 10$ schoolchildren $(10.2$ years $(8.0-13.8))$ and $2 / 7$ adults (28 years $(18-33))$ whereas sensitization to Cor a 9 was observed in 16/20 preschoolchildren, 7/10 schoolchildren and $3 / 7$ adults. A minority of $13 / 37$ and $5 / 37$ was sensitized to Cor a 11 and Cor a 8. Combining of Cor a 14 and Cor a 9 enables us to correctly diagnose respectively $100 \%, 80 \%$ and $43 \%$ of systemic reactions in preschool-, schoolchildren and adults. In contrast sensitization to
Cor a 1.04 was generally associated with OAS, IgE reactivity to Cor a 1.04 was observed in respectively 6/7, 8/9 and $22 / 22$ of preschool- , schoolchildren and adults. Sensitization to Cor a 14 was seen in two patients with OAS, although these sIgE levels to Cor a 14 were significantly lower. Twenty-one percent of the infants with AD showed Cor a 14 sensitization, whereas $4 / 14$ and $1 / 14$ showed IgE reactivity to Cor a 9 and Cor a 11 .

\section{Conclusion}

Quantification of Cor a 14 can be of great value in hazelnut allergy diagnosis. Sensitization to Cor a 14 predominantly occurs in pre- and schoolchildren with severe hazelnut allergy and can have early onset ( $<1$ year of age).

\begin{abstract}
Authors' details
${ }^{1}$ Department of Immunology-Allergology-Rheumatology, Faculty of Medicine and Health Sciences, University of Antwerp, Antwerpen, Belgium

2Department of Immunology-Allergology-Rheumatology, Antwerp University Hospital, Antwerpen, Belgium. ${ }^{3}$ Department of Pediatrics, Antwerp University Hospital, Antwerpen, Belgium.
\end{abstract}

Published: 28 February 2014

doi:10.1186/2045-7022-4-S1-011

Cite this article as: Faber et al:: 011 - Cor a 14: the missing link in the molecular diagnosis of hazelnut allergy? Clinical and Translational Allergy 2014 4(Suppl 1):011. 